\title{
An Updated Meta-analysis on the Risk of Urologic Cancer in Patients with Systemic Lupus Erythematosus
}

\author{
Jina Yeo, $\mathrm{MD}^{1 *}$; Min-Seok Seo, $\mathrm{MD}^{2,3 *}$; In Cheol Hwang, $\mathrm{PhD}^{4 *}$; Jae-Yong Shim, $\mathrm{PhD}^{3}$ \\ ${ }^{1}$ Rheumatology Division, Department of Internal Medicine, Gachon University Gil Medical Center, Incheon, Republic of Korea \\ ${ }^{2}$ Department of Family Medicine, Incheon St. Mary's Hospital, Incheon, Republic of Korea \\ ${ }^{3}$ Yonsei University Graduate School of Medicine, Seoul, Republic of Korea \\ ${ }^{4}$ Department of Family Medicine, Gil Medical Center, Gachon University College of Medicine, Incheon, Republic of Korea
}

\begin{abstract}
Background: The risk of urologic cancers in patients with systemic lupus erythematosus (SLE) remains uncertain. We investigated the association between SLE and incident urologic cancers through a systematic review and meta-analysis.

Methods: We searched the PubMed, EMBASE, and the Cochrane Library to identify articles that recorded prostate, bladder, or kidney cancers in SLE patients from inception to August 31, 2018. We included observational, case-control, or cohort studies with no language restriction. Two investigators screened and extracted the data independently.

Results: Fourteen cohort studies with 83,860 SLE patients were finally analyzed. Overall, SLE patients were at increased risk of bladder cancer (hazard ratio [HR], 1.92; 95\% confidence interval [Cl], 1.15-3.21) but not of prostate or kidney cancer. However, subgroup analyses showed a reduced risk of prostate cancer in <10-year follow-up studies (HR, $0.68 ; 95 \% \mathrm{Cl}, 0.51-0.89)$ and an elevated risk of kidney cancer in patients with SLE in Western studies (HR, 2.00; 95\% Cl, 1.02-3.92), community-based studies (HR, 4.54; 95\% Cl, 2.17-9.52), prospective studies (HR, 6.84; 95\% Cl, 2.71-17.26), <10-year follow-up studies (HR, 1.88; 95\% $\mathrm{Cl}, 1.38-2.57)$, and low-quality studies (HR, 2.05; 95\% Cl, 1.50-2.80).

Conclusion: This study indicates that SLE increases the risk of bladder cancer but not prostate or kidney cancer. Well-designed long-term studies are required to confirm these associations.

Keywords: Bladder cancer, Kidney cancer, Meta-analysis, Prostate cancer, Systemic lupus erythematosus

Cite this article as: Yeo J, Seo MS, Hwang IC, Shim JY. An updated meta-analysis on the risk of urologic cancer in patients with systemic lupus erythematosus. Arch Iran Med. 2020;23(9):614-620. doi: 10.34172/aim.2020.72.
\end{abstract}

Received: July 29, 2019, Accepted: April 22, 2020, ePublished: September 1, 2020

\section{Introduction}

Systemic lupus erythematosus (SLE) affects virtually every organ and causes diverse clinical manifestations and laboratory findings. ${ }^{1}$ SLE is usually treated with systemic steroids and/or immune suppressants. ${ }^{2}$ Recently, biologic agents have been used to increase the survival rate of SLE patients. ${ }^{3}$ However, associated conditions (e.g., dyslipidemia, diabetes mellitus, and osteoporosis), and particularly malignancies, still play a crucial role in the long-term outcomes of SLE patients. ${ }^{4}$ Several studies have indicated that SLE patients are at increased risk for malignant tumors caused by a decline of the intrinsic immune system and exposure to cytotoxic agents. ${ }^{5,6}$ The association between SLE and malignant tumors is still not fully understood, and cancer surveillance for patients with SLE is the same as that for the general population. ${ }^{\text {? }}$

Urologic cancers are relatively common. Prostate cancer remains the second most frequent cancer and the fifth leading cause of male cancer death, accounting for $7.1 \%$ of all new cases of cancer and 3.8\% of all cancer deaths worldwide in 2018. Bladder cancer ranks as the tenth most common cancer, with approximately 549000 new cases and 200000 deaths worldwide in 2018. ${ }^{8}$ Kidney cancer accounted for nearly 403000 new cases and 175000 deaths in 2018.

Cammarata et al reported an association between SLE and lymphoma in 1963. ${ }^{9}$ Since then, the relationship between SLE and malignancies, including urologic cancers, has been studied. According to previous researches, some potential mechanisms in the development of urologic cancers, especially prostate cancer, are related to hormonal effects and cytokine pathways. ${ }^{10,11}$

Until now, several meta-analyses have shown inconclusive results concerning incident urologic cancers in SLE patients. ${ }^{12-15}$ A decreased risk of prostate cancer was shown in Huang's study, while Cao et al and Mao et al failed to show a decrease. ${ }^{12-14}$ With respect to bladder cancer, only Cao et al demonstrated that SLE patients were at increased risk of bladder cancer. ${ }^{12}$ Additionally, Huang et al. and Mao et al. found higher risk of kidney cancer among SLE patients, ${ }^{13,14}$ while Cao et al found that SLE had no effect on the risk of kidney cancer. ${ }^{12}$ Recently, 
Song et al reported lower risk of prostate cancer and higher risk of bladder and kidney cancer in SLE patients, ${ }^{15}$ but their study has a critical problem with duplicate data. Therefore, to elucidate the association between SLE and urologic malignancy, we conducted a meta-analysis using published studies without duplicate data.

\section{Materials and Methods}

Data Sources and Literature Search

A systematic review and meta-analysis were performed to evaluate incident urologic cancer among SLE patients. We followed the guideline of Preferred Reporting Items for Systematic Reviews and Meta-Analyses (PRISMA) statement. ${ }^{16}$ For this systematic review, we identified studies reporting the incidence of urologic cancers in SLE patients. Two investigators (J.Y. and M.S.S) independently searched the PubMed, EMBASE, and the Cochrane Library databases for articles published from inception to August 31, 2018 that reported on the relationships between SLE and urologic cancers. The bibliography of the included papers was searched manually for additional studies. We used the following search terms: cancer, carcinoma, neoplasm, malignancy, malignancies, tumor, or tumour with systemic lupus erythematosus, autoimmune disease, rheumatic disease, or SLE. No limits were applied for language.

\section{Eligibility Criteria and Study Selection}

Eligible studies fulfilled the following inclusion criteria: 1) observational, case-control, or cohort study; 2) SLE as one of exposures; 3) urologic cancer (prostate, bladder, or kidney) as one of outcomes; 4) general population as control; and 5) providing standardized incidence rate, hazard ratio (HR), relative risk, or odds ratio and the corresponding 95\% confidence intervals (CIs). The exclusion criteria were: 1) reviews, editorials, letters, case reports or expert opinions; or 2) duplicated publications or overlapping study populations. Two investigators independently identified articles that met the eligibility criteria through screening all titles and abstracts, followed by reviews of full-text. When there were duplicates from the same database or overlapping populations, we chose the most appropriate study for inclusion in our analysis. Any inconsistency in opinions between the two investigators was resolved by an independent investigator (I.C.H).

Data Collection and Quality Assessment

Two investigators (J.Y. and M.S.S) collected the relevant data independently. The extracted data included: first author, year published, country, data source, gender, design type, follow-up periods, observed/estimated number, estimates and 95\% CIs, and adjusted covariates. We used a Newcastle-Ottawa Scale (NOS) to assess the methodological quality of study. ${ }^{17}$ Based on the median values in our analysis, high quality studies were defined as those with an NOS score of 7 or more. Any disagreements between two investigators were solved through discussion and consensus with all authors.

\section{Statistical Analysis}

Urologic cancer risk was evaluated using pooled HRs with 95\% CIs, which were calculated cumulatively across the selected publication. We also performed subgroup analyses by ethnicity, study design, data source, follow-up period, and study quality. In contrast to the other two cancers, prostate cancer was analyzed only for male subjects. Between-study heterogeneity was estimated by Higgins $\mathrm{I}^{2}(0-100 \%) .{ }^{18}$ When heterogeneity was substantial $\left(I^{2}\right.$ $\geq 50 \%$ ), a random (DerSimonian-Laird) effect model was applied. ${ }^{19}$ Furthermore, we performed a sensitivity analysis to reflect the impact of different decisions on outcomes by excluding specific study. We checked for publication bias using Begg's rank correlation. We performed statistical analyses using Stata v12.1 (Stata Corp, College Station, TX, USA).

\section{Results}

Search and Characteristics

A flowchart of the study identification procedure is shown in Figure 1. A total of 1,011 articles were initially screened through a systematic search. We excluded duplicate publications $(n=430)$ and papers that appeared irrelevant based on the title $(n=447)$. After screening the abstracts, we excluded 117 studies due to the following reasons: not original articles $(\mathrm{n}=36)$, not focusing on cancer risk $(\mathrm{n}=$ $23)$, and not including urologic cancers $(\mathrm{n}=58)$. We then conducted a full-text review of 17 articles that fulfilled our eligibility criteria. Six papers were further excluded because of overlapping populations and three papers were added after conducting a manual literature search. Thus,

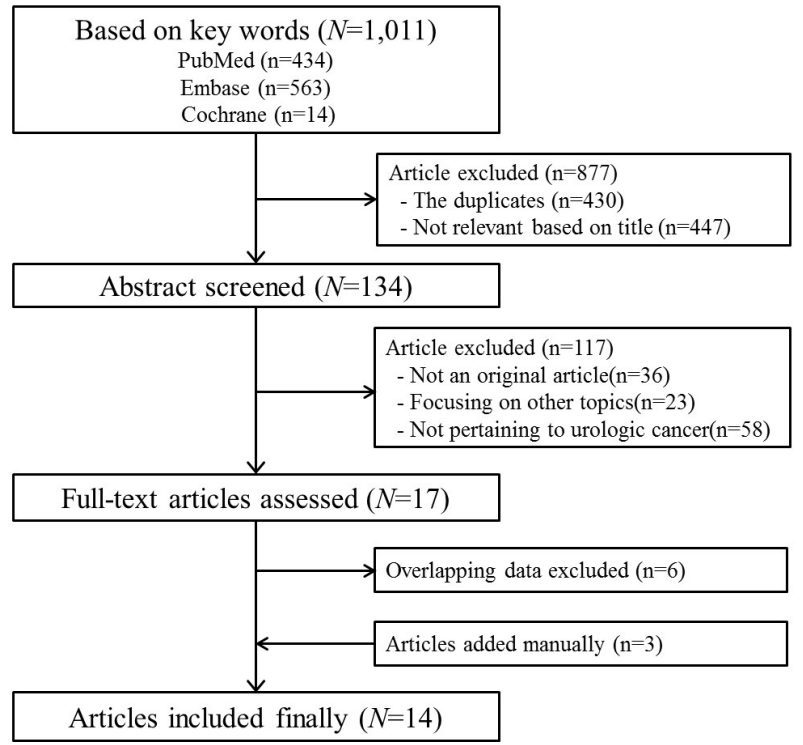

Figure 1. Flow Chart for Searching Relevant Articles 
14 articles were finally included in the analysis.

Detailed information about the studies are presented in Table 1. Our meta-analysis included a total of 83,860 patients with SLE. The publication period was from 1996 to 2018. Eight studies were conducted in Europe, two in Asia, and three in North America. There was also one multinational study from North America, Europe, and Korea. Eleven of the studies were retrospective, and three were prospective. Nine of the studies were hospital-based, while the other five were population-based. Supplementary Table 1 shows the methodological quality of studies. The mean NOS score among the 14 studies was 6.28. Six of the studies were classified as high quality.

\section{Urologic Cancers in SLE}

There were 10 studies providing HRs for prostate cancers. The pooled HR for prostate cancer among patients with SLE was 0.92 (95\% CI, 0.65-1.30; $\mathrm{I}^{2}=74.7 \%$; Figure 2), suggesting that SLE was not a significant risk factor for prostate cancer. However, subgroup analyses restricted by follow-up duration showed that patients with SLE had a reduced risk of prostate cancer in <10-year follow-up studies within the fixed-effects model (HR, 0.68; 95\% CI, 0.51-0.89; Table 2).

For bladder cancer, analysis of 10 studies revealed that the overall HR was 1.92 (95\% CI, 1.15-3.21; $\mathrm{I}^{2}=80.2 \%$; Figure 2). Our results indicated that SLE was associated with higher risk of bladder cancer. Similarly, sensitivity analysis by excluding Kang's study presented significant association (HR, 1.46; 95\% CI, 1.01-2.11; $\mathrm{I}^{2}=59.5 \%$, random-effects model). In the subgroup analyses, the risk of bladder cancer was also higher in SLE patients in community-based studies (HR, 3.14; 95\% CI, 1.059.41), retrospective studies (HR, 1.97; 95\% CI, 1.05-
3.70), and $\geq 10$-year follow-up studies (HR, 2.38; 95\% CI, 1.76-3.23) (Table 2).

Finally, kidney cancer was analyzed in eight studies. They failed to indicate a significant association between SLE and kidney cancer (HR, 1.72; 95\% CI, 0.94-3.14; $I^{2}=62.7 \%$; Figure 2). The regional subgroup analysis revealed that Western (North American and European) studies conferred a significantly higher risk of kidney cancer within the random-effects model (HR, 2.00; 95\% CI, 1.02-3.92). When we stratified subgroup analysis by some characteristics, we found that the risk of kidney cancer was also higher in SLE patients among communitybased studies (HR, 4.54; 95\% CI, 2.17-9.52), prospective studies (HR, 6.84; 95\% CI, 2.71-17.26), <10-year followup studies (HR, 1.88; 95\% CI, 1.38-2.57), and lowquality studies (HR, 2.05; 95\% CI, 1.50-2.80) within the fixed-effects model.

There was no marked asymmetry in the funnel plot (Figure 3). No publication biases were noted from the Begg's $(P=0.621)$ and Egger's tests $(P=0.733)$.

\section{Discussion}

A number of studies have examined the association between SLE and cancers and found an increased overall cancer risk in patients with SLE. ${ }^{20-22}$ There is accumulating evidence that the risks of non-Hodgkin's lymphoma, leukemia, cancer of the vulva, and lung cancer are increased in patients with SLE, whereas results have been inconsistent for some other solid cancers, including urologic cancers. ${ }^{23,24}$ Several meta-analyses have attempted to clarify the relationship between SLE and urologic cancers, but the results have been mixed. ${ }^{12-15}$ We identified a critical error in the data selection for the most recent meta-study by Song et al, ${ }^{15}$ who included duplicate data from three studies. ${ }^{21,25-26} \mathrm{On}$

Table 1. Characteristics of the Included Studies

\begin{tabular}{|c|c|c|c|c|c|c|c|c|}
\hline Study & Year & Country & Data source & Design & $\begin{array}{l}\text { SLE (Total } n / \\
\text { Female \%) }\end{array}$ & $\begin{array}{l}\text { Follow-up } \\
\qquad(y)\end{array}$ & $\begin{array}{l}\text { Adjusted Variables other than Age } \\
\text { and Sex }\end{array}$ & $\begin{array}{l}\text { Quality Score } \\
(0-9)\end{array}$ \\
\hline Abu-Shakra ${ }^{53}$ & 1996 & Canada & Hospital & Prospective & $724 / 86.6$ & 10 & & 7 \\
\hline Mellemkjaer ${ }^{34}$ & 1997 & Denmark & Hospital & Retrospective & $1585 / 83$ & 6.8 & & 6 \\
\hline Cibere $^{22}$ & 2001 & Canada & Hospital & Retrospective & $297 / 84$ & 12 & & 7 \\
\hline Ragnarsson ${ }^{54}$ & 2003 & Iceland & Nationwide & Retrospective & $238 / 89.5$ & 12.8 & & 7 \\
\hline Tarr ${ }^{55}$ & 2007 & Hungary & Hospital & Retrospective & $860 / 89.7$ & 16.5 & & 6 \\
\hline Parikh-Patel ${ }^{24}$ & 2008 & USA & Statewide & Retrospective & $30478 / 89$ & 5.1 & Race & 7 \\
\hline Kang ${ }^{32}$ & 2010 & Korea & Hospital & Retrospective & $914 / 100$ & 6.2 & & 6 \\
\hline Dreyer $^{33}$ & 2011 & Denmark & Hospital & Retrospective & $576 / 88$ & 13.2 & & 5 \\
\hline Bernatsky ${ }^{23}$ & 2013 & International & Hospital & Prospective & $16409 / 90$ & 7.4 & & 6 \\
\hline Dey $^{27}$ & 2013 & UK & Hospital & Retrospective & 595/NR & 14.7 & & 6 \\
\hline $\operatorname{Liu}^{29}$ & 2013 & Sweden & Nationwide & Retrospective & $7624 / \mathrm{NR}$ & 11.3 & Obesity, region, alcohol, smoking & 7 \\
\hline $\operatorname{Rees}^{30}$ & 2016 & UK & Nationwide & Retrospective & 7732/85.8 & 8.4 & $\begin{array}{l}\text { Alcohol, smoking, hypertension, } \\
\text { BMI, Charlson index score, } \\
\text { hyperlipidemia, Prednisolone }\end{array}$ & 6 \\
\hline $\mathrm{Yu}^{28}$ & 2016 & Taiwan & Nationwide & Retrospective & $15623 / 87.6$ & 7.9 & & 5 \\
\hline Tallbacka ${ }^{56}$ & 2018 & Finland & Hospital & Prospective & 205/88.78 & 25.7 & & 7 \\
\hline
\end{tabular}

BMI, body mass index; NR, not reported; SLE, systemic lupus erythematosus. 


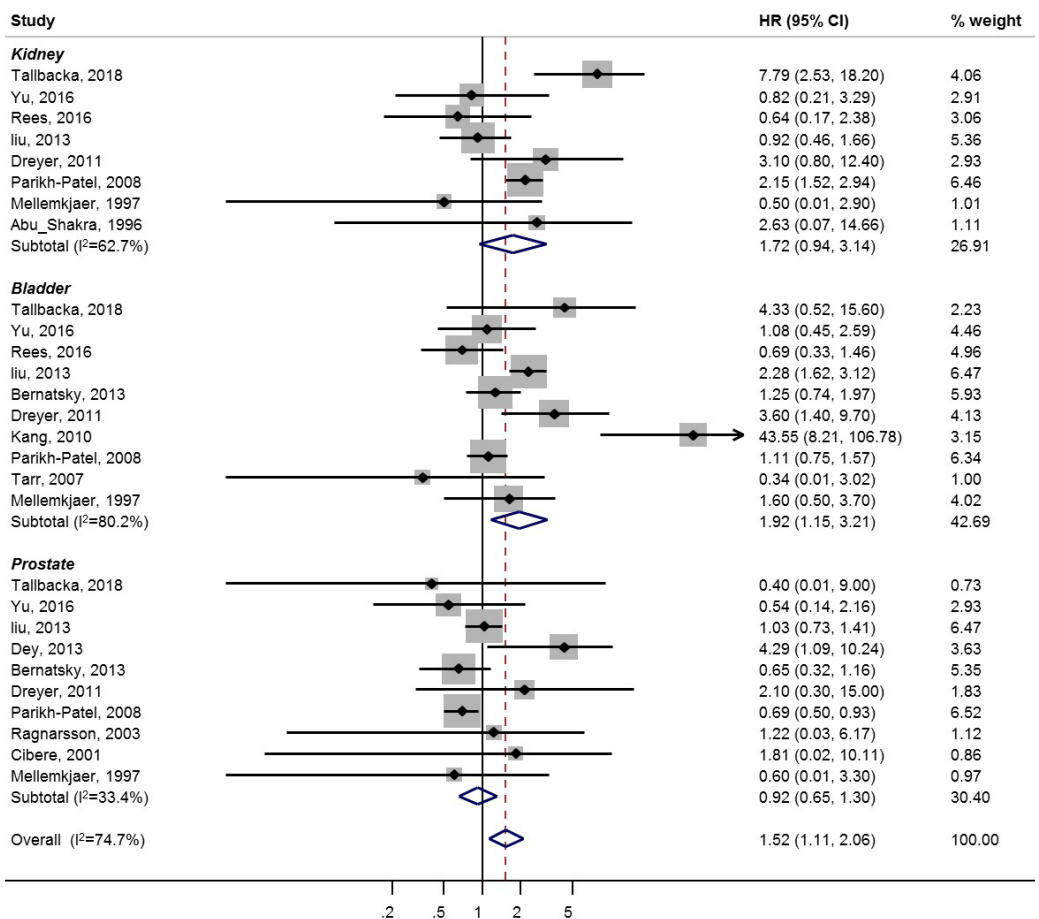

Figure 2. Forest Plot of Site-Specific Urologic Cancer Risk in SLE Patients. SLE, systemic lupus erythematosus; HR, hazard ratio; Cl, confidence interval.

the other hand, some studies used more updated data. ${ }^{23,27-}$ ${ }^{28}$ Another crucial problem with previous meta-analyses of SLE and urological cancer risk is that some of the metaanalyses missed some principal studies. We included Liu's study instead of Bjornadal's study in our meta-analysis, because the former used more inclusive data. ${ }^{20,29}$ We also included Rees's study. ${ }^{30}$ Complementing some of the limitations of Song's meta-analysis, ${ }^{15}$ our study provides more specific estimates from unduplicated and recent data.

In this study, bladder cancer was more commonly developed in SLE patients compared with the general population. Previous meta-studies have shown inconsistent results. ${ }^{12-15}$ However, some researchers suggested that the high risk for bladder cancer in SLE patients might be due to lupus cystitis, viral infection, or SLE treatment such as cyclophosphamide. ${ }^{31-33}$ In addition, stratified analyses suggested several factors (i.e., data sources, study design and follow-up duration) as potential mediators for the relationships between SLE and bladder cancer.

On the other hand, the association of SLE with prostate

Table 2. Risk of Urologic Cancers in SLE Patients

\begin{tabular}{|c|c|c|c|c|c|c|c|c|c|}
\hline & \multicolumn{3}{|c|}{ Kidney } & \multicolumn{3}{|c|}{ Bladder } & \multicolumn{3}{|c|}{ Prostate } \\
\hline & $n$ & $\mathrm{HR}(95 \% \mathrm{Cl})$ & Model & $n$ & $\mathrm{HR}(95 \% \mathrm{CI})$ & Model & $n$ & HR $(95 \% \mathrm{CI})$ & Model \\
\hline Overall & 8 & $1.72(0.94-3.14)$ & Random & 10 & $1.92(1.15-3.21)$ & Random & 10 & $0.86(0.70-1.05)$ & Fixed \\
\hline \multicolumn{10}{|l|}{ Ethnicity } \\
\hline Asian & 2 & $0.75(0.22-2.57)$ & Fixed & 3 & $4.03(0.52-31.26)$ & Random & 2 & $0.55(0.16-1.90)$ & Fixed \\
\hline Western & 6 & $2.00(1.02-3.92)$ & Random & 7 & $1.50(0.96-2.35)$ & Random & 8 & $0.87(0.71-1.07)$ & Fixed \\
\hline \multicolumn{10}{|l|}{ Data source } \\
\hline Nationwide & 4 & $4.54(2.17-9.52)$ & Fixed & 6 & $3.14(1.05-9.41)$ & Random & 5 & $1.45(0.50-4.23)$ & Random \\
\hline Hospital-based & 4 & $1.19(0.61-2.29)$ & Random & 4 & $1.25(0.72-2.15)$ & Random & 5 & $0.82(0.66-1.03)$ & Fixed \\
\hline \multicolumn{10}{|l|}{ Design } \\
\hline Prospective & 2 & $6.84(2.71-17.26)$ & Fixed & 2 & $1.37(0.86-2.20)$ & Fixed & 2 & $0.64(0.34-1.20)$ & Fixed \\
\hline Retrospective & 6 & $1.32(0.75-2.30)$ & Random & 8 & $1.97(1.05-3.70)$ & Random & 8 & $0.89(0.72-1.10)$ & Fixed \\
\hline \multicolumn{10}{|c|}{ Follow-up duration } \\
\hline$\geq 10$ years & 4 & $2.67(0.77-9.27)$ & Random & 4 & $2.38(1.76-3.23)$ & Fixed & 6 & $1.17(0.86-1.59)$ & Fixed \\
\hline$<10$ years & 4 & $1.88(1.38-2.57)$ & Fixed & 6 & $1.75(0.85-3.56)$ & Random & 4 & $0.68(0.51-0.89)$ & Fixed \\
\hline \multicolumn{10}{|l|}{ Study quality } \\
\hline High (NOS $\geq 7$ ) & 4 & $1.80(0.49-6.54)$ & Random & 4 & $1.50(0.82-2.76)$ & Random & 4 & $0.93(0.70-1.25)$ & Fixed \\
\hline Low $($ NOS < $>$ ) & 4 & $2.05(1.50-2.80)$ & Fixed & 6 & $2.40(0.89-6.49)$ & Random & 6 & $1.18(0.52-2.70)$ & Random \\
\hline
\end{tabular}

SLE, systemic lupus erythematosus; HR, hazard ratio; Cl, confidence interval; NOS, Newcastle-Ottawa Scale. 


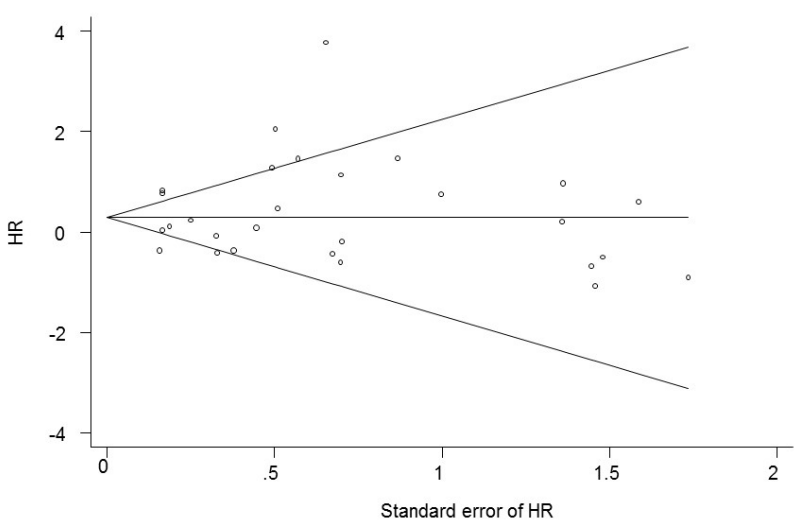

Figure 3. Funnel Plot Using a Begg's Rank Correlation among the Included Studies. HR, hazard ratio.

cancer remained insignificant. An early meta-analysis reported low risk of prostate cancer in SLE patients; ${ }^{13}$ but two subsequent meta-studies did not show a significant relationship between SLE and reduced risk of prostate cancer. ${ }^{12,14}$ Huang's study was hampered by missing data, however, whereas Mao's study contained duplicate data. To clarify that inconsistency, we used the most recent available data without duplicates. There are some clinical considerations that might shed some light on our result. First, SLE is a female-dominant disease, ${ }^{34}$ but prostate cancer only occurs in males, so there might be insufficient data to examine the incident prostate cancer among patients with SLE. Second, prostate cancer has peak incidence in males 65 years of age or older. ${ }^{35}$ Interestingly, our subgroup analysis presented a low risk of prostate cancer among studies with $<10$-year follow-up period. It may be attributed to hormonal effect, which is remarkable in patients at early stage of SLE, a relatively large proportion of whom have severe symptoms. Given the high disease activity of SLE correlated to hypoandrogenism, ${ }^{10,36}$ we assumed that patients at early stage SLE have high disease activity but low testosterone level, leading to lower risk for developing prostate cancer.

For kidney cancer, we did not find an overall significant association, in contrast to two previous meta-studies. ${ }^{14,15}$ In our subgroup analyses, however, the risk of kidney cancer was increased in Western SLE patients but not in Asian SLE patients. The difference in the risk of kidney cancer by ethnicity may be explained partly by genetic backgrounds which influence immunity and tumor genesis. ${ }^{37}$ It is well known that the incidence of kidney cancer in the general population is higher in Europe and North America compared to Asia. ${ }^{38}$ Considering the higher incidence of kidney cancer in Western populations, we suggest that Western SLE patients should be attentively monitored for the development of kidney cancer.

We found high risk for kidney cancer among nationwide studies but not in hospital-based studies. The nationwide data might have an advantage in that they included the actual primary diagnosis, whereas the hospital-based data were secondary to the identification of cases. Despite efforts to minimize data errors, even the nationwide data may contain coding and keying errors. The use of hospitalbased data requires careful considerations to account for imperfect ascertainment of disease status, ${ }^{39}$ because hospital-based studies use administrative data and various algorithms to identify SLE, which may result in reduced quality of diagnoses.

We found that SLE patients had a high risk for kidney cancer among studies with $<10$ years of followup. That might be partly explained by the intensive use of immunosuppressive agents during the early stage of SLE. ${ }^{40}$ Most studies, including our meta-analysis, did not consider the use of immunosuppressants. The cumulative effects of immunosuppressants are another important consideration. ${ }^{41}$ Studies with long follow-up reflecting long-term use of immunosuppressive drugs are required to clarify our results.

Although the relationship between SLE and urologic cancers remains unclear, there are some plausible hypotheses that might explain the elevated risk of urologic cancers in patients with SLE. First, the chronic inflammation in SLE can influence the development of urologic cancers. Chronic inflammation enhances the production of inflammatory cytokines, which might increase the development and growth of cancer. ${ }^{42}$ In urologic cancers, chronic inflammation promotes tumor evasion and progression. ${ }^{43-45}$ Second, SLE per se decreases immune-cell function, which results in dysregulation of the immune system. Generally, a healthy immune system removes aberrantly replicating cells to prevent malignancies, whereas this process may be impaired in SLE patients. ${ }^{46}$ In particular, the use of immunosuppressive agents has an influence on the risk of cancer in SLE patients. ${ }^{47}$ Several case series suggested that immunosuppressive agents increased the risk of bladder cancer in patients with SLE. ${ }^{48-}$ ${ }^{50}$ Bernasky et al reported that immunosuppressive therapy in patients with SLE increased the bladder cancer risk by $25 \% .{ }^{51}$ SLE patients who receive immunosuppressive therapy may be exposed to harmful side effects and immune impairment, which may lead to cell damage and promote tumor genesis. ${ }^{32}$ Immunosuppressants damage cells directly, and the immune-compromised state impairs the body's ability to repair destroyed or damaged cells and cellular DNA. ${ }^{52}$

This study has several limitations. First, there was high heterogeneity in the rates of kidney cancer and bladder cancer across the studies. This might hinder accurate estimation of the risks associated with SLE; however, we conducted subgroup analyses to minimize the confounding factors that contributed to heterogeneity. Second, we could not evaluate the cumulative effects of exposure to immune suppressants and disease activity because of lack 
of studies that included those data. Third, we could not assess confounding variables, such as smoking, alcohol history, and chronic diseases.

Despite the limitations, our results strongly indicate that SLE increases the risk of bladder cancer, but not those of prostate cancer and kidney cancer. Our results might be helpful to guide physicians that care for patients with SLE and urologic cancers. Our results also add to the body of evidence that can be used to develop better strategies to screen for and follow-up on incident urologic cancers in SLE patients. More well-designed prospective studies and more experimental data are needed to elucidate the underlying mechanisms of the associations between SLE and urologic cancers.

\section{Authors' Contribution}

JY, MSS, and ICH made the conceptual ideas and wrote the manuscript. ICH worked out almost all the analytical details and coordinated the study. All authors searched literatures, interpreted the results, and approved the final version of manuscript.

\section{Conflict of Interest Disclosures}

None.

\section{Ethical Statement}

This meta-analysis is exempt from ethics approval because we only collected and synthesized data from previous studies in which informed consent has already been obtained.

\section{Funding}

None.

\section{References}

1. D'Cruz DP, Khamashta MA, Hughes GR. Systemic lupus erythematosus. Lancet. 2007;369(9561):587-96. doi: 10.1016/S0140-6736(07)60279-7.

2. Zintzaras E, Voulgarelis M, Moutsopoulos HM. The risk of lymphoma development in autoimmune diseases: a metaanalysis. Arch Intern Med. 2005;165(20):2337-44. doi: 10.1001/archinte.165.20.2337.

3. Amissah-Arthur MB, Gordon C. Contemporary treatment of systemic lupus erythematosus: an update for clinicians. Ther Adv Chronic Dis. 2010;1(4):163-75. doi: 10.1177/2040622310380100.

4. Bertsias G, loannidis JP, Boletis J, Bombardieri S, Cervera R, Dostal C, et al. EULAR recommendations for the management of systemic lupus erythematosus. Report of a Task Force of the EULAR Standing Committee for International Clinical Studies Including Therapeutics. Ann Rheum Dis. 2008;67(2):195-205. doi: 10.1136/ard.2007.070367.

5. Bernatsky S, Clarke A, Ramsey-Goldman R. Malignancy and systemic lupus erythematosus. Curr Rheumatol Rep. 2002;4(4):351-8. doi: 10.1007/s11926-002-0045-6.

6. Kiss E, Kovacs L, Szodoray P. Malignancies in systemic lupus erythematosus. Autoimmun Rev. 2010;9(4):195-9. doi: 10.1016/j.autrev.2009.07.004.

7. Chang SH, Park JK, Lee YJ, Yang JA, Lee EY, Song YW, et al. Comparison of cancer incidence among patients with rheumatic disease: a retrospective cohort study. Arthritis Res Ther. 2014;16(4):428. doi: 10.1186/s13075-014-0428-x.

8. Bray F, Ferlay J, Soerjomataram I, Siegel RL, Torre LA, Jemal A. Global cancer statistics 2018: GLOBOCAN estimates of incidence and mortality worldwide for 36 cancers in 185 countries. CA Cancer J Clin. 2018;68(6):394-424. doi: 10.3322/caac. 21492.

9. Cammarata RJ, Rodnan GP, Jensen WN. Systemic rheumatic disease and malignant lymphoma. Arch Intern Med. 1963; 111:330-7. doi: 10.1001/archinte.1963.03620270056009.

10. Mok CC, Lau CS. Profile of sex hormones in male patients with systemic lupus erythematosus. Lupus. 2000;9(4):252-7. doi: 10.1191/096120300680198926.

11. Foster CS, Dodson AR, Ambroisine L, Fisher G, Moller H, Clark J, et al. Hsp-27 expression at diagnosis predicts poor clinical outcome in prostate cancer independent of ETSgene rearrangement. Br J Cancer. 2009;101(7):1137-44. doi: 10.1038/sj.bjc.6605227.

12. Cao L, Tong H, Xu G, Liu P, Meng H, Wang J, et al. Systemic lupus erythematous and malignancy risk: a meta-analysis. PLoS One. 2015;10(4):e0122964. doi: 10.1371/journal. pone. 0122964

13. Huang HB, Jiang SC, Han J, Cheng QS, Dong CB, Pan CM. A systematic review of the epidemiological literature on the risk of urological cancers in systemic lupus erythematosus. J Cancer Res Clin Oncol. 2014;140(7):1067-73. doi: 10.1007/ s00432-014-1604-8.

14. Mao S, Shen H, Zhang J. Systemic lupus erythematosus and malignancies risk. J Cancer Res Clin Oncol. 2016;142(1):25362. doi: 10.1007/s00432-015-2032-0.

15. Song L, Wang Y, Zhang J, Song N, Xu X, Lu Y. The risks of cancer development in systemic lupus erythematosus (SLE) patients: a systematic review and meta-analysis. Arthritis Res Ther. 2018;20(1):270-82. doi: 10.1186/s13075-018-1760-3.

16. Moher D, Liberati A, Tetzlaff J, Altman DG, PRISMA Group. Preferred reporting items for systematic reviews and metaanalyses: the PRISMA statement. BMJ 2009;339:b2535. doi: 10.1136/bmj.b2535.

17. Stang A. Critical evaluation of the Newcastle-Ottawa scale for the assessment of the quality of nonrandomized studies in meta-analyses. Eur J Epidemiol. 2010;25(9):603-5. doi: 10.1007/s10654-010-9491-z.

18. Higgins JP, Thompson SG. Quantifying heterogeneity in a meta-analysis. Stat Med. 2002;21(11):1539-58. doi: 10.1002/ sim. 1186.

19. DerSimonian R, Laird N. Meta-analysis in clinical trials Control Clin Trials. 1986;7(3):177-88. doi: 10.1016/01972456(86)90046-2.

20. Bjornadal L, Lofstrom B, Yin L, Lundberg IE, Ekbom A. Increased cancer incidence in a Swedish cohort of patients with systemic lupus erythematosus. Scand J Rheumatol. 2002;31(2):66-71. doi: 10.1080/03009740252937568.

21. Chen YJ, Chang YT, Wang CB, Wu CY. Malignancy in systemic lupus erythematosus: a nationwide cohort study in Taiwan. Am J Med. 2010;123(12):1150.e1-6. doi: 10.1016/j. amjmed.2010.08.006.

22. Cibere J, Sibley J, Haga M. Systemic lupus erythematosus and the risk of malignancy. Lupus. 2001;10(6):394-400. doi: 10.1191/096120301678646128.

23. Bernatsky S, Ramsey-Goldman R, Labrecque J, Joseph L, Boivin JF, Petri M, et al. Cancer risk in systemic lupus: an updated international multi-centre cohort study. J Autoimmun. 2013;42:130-5. doi: 10.1016/j.jaut.2012.12.009.

24. Parikh-Patel $A$, White RH, Allen M, Cress R. Cancer risk in a cohort of patients with systemic lupus erythematosus (SLE) in California. Cancer Causes Control. 2008;19(8):887-94. doi: 10.1007/s10552-008-9151-8.

25. Bernatsky S, Boivin JF, Joseph L, Rajan R, Zoma A, Manzi S, et al. An international cohort study of cancer in systemic lupus erythematosus. Arthritis Rheum. 2005;52(5):1481-90. doi: 10.1002/art.21029.

26. Sultan SM, loannou $Y$, Isenberg DA. Is there an association of malignancy with systemic lupus erythematosus? An analysis of 276 patients under long-term review. Rheumatology (Oxford). 2000;39(10):1147-52. doi: 10.1093/ rheumatology/39.10.1147. 
27. Dey D, Kenu E, Isenberg DA. Cancer complicating systemic lupus erythematosus--a dichotomy emerging from a nested case-control study. Lupus. 2013;22(9):919-27. doi: 10.1177/0961203313497118.

28. Yu KH, Kuo CF, Chou IJ, Chiou MJ, See LC. Risk of endstage renal disease in systemic lupus erythematosus patients: a nationwide population-based study. Int J Rheum Dis. 2016;19(11):1175-82. doi: 10.1111/1756-185X.12828.

29. Liu X, Ji J, Forsti A, Sundquist K, Sundquist J, Hemminki K. Autoimmune disease and subsequent urological cancer. J Urol. 2013;189(6):2262-8. doi: 10.1016/j.juro.2012.12.014.

30. Rees F, Doherty M, Grainge MJ, Lanyon P, Zhang W. The worldwide incidence and prevalence of systemic lupus erythematosus: a systematic review of epidemiological studies. Rheumatology (Oxford). 2017;56(11):1945-61. doi: 10.1093/rheumatology/kex260.

31. Akagi T, Fujita S, MukaiT, Fujita M, Morita Y. Bladder carcinoma in situ in a patient with Lupus cystitis. Mod Rheumatol Case Rep. 2019;3(1):20-3. doi: 10.1080/24725625.2018.1519170.

32. Kang KY, Kim HO, Yoon HS, Lee J, Lee WC, Ko HJ, et al. Incidence of cancer among female patients with systemic lupus erythematosus in Korea. Clin Rheumatol. 2010;29(4):381-8. doi: 10.1007/s10067-009-1332-7.

33. Dreyer L, Faurschou M, Mogensen M, Jacobsen S. High incidence of potentially virus-induced malignancies in systemic lupus erythematosus: a long-term followup study in a Danish cohort. Arthritis Rheum. 2011;63(10):3032-7. doi: 10.1002/art.30483.

34. Mellemkjaer L, Andersen V, Linet MS, Gridley G, Hoover R, Olsen JH. Non-Hodgkin's lymphoma and other cancers among a cohort of patients with systemic lupus erythematosus. Arthritis Rheum. 1997;40(4):761-8. doi: 10.1002/art.1780400424.

35. Patel AR, Klein EA. Risk factors for prostate cancer. Nat Clin Pract Urol. 2009;6(2):87-95. doi: 10.1038/ncpuro1290.

36. Arnaud L, Nordin A, Lundholm H, Svenungsson E, Hellbacher E, Wikner J, et al. Effect of corticosteroids and cyclophosphamide on sex hormone profiles in male patients with systemic lupus erythematosus or systemic sclerosis. Arthritis Rheumatol. 2017;69(6):1272-9. doi: 10.1002/ art. 40057 .

37. Hoi A. Asian lupus in a multi-ethnic society: what can be learnt? Int J Rheum Dis. 2015;18(2):113-6. doi: 10.1111/1756$185 X .12497$.

38. Scelo G, Larose TL. Epidemiology and Risk Factors for Kidney Cancer. J Clin Oncol. 2018:36(36):3574-81. doi: 10.1200/ JCO.2018.79.1905.

39. Bernatsky S, Joseph L, Pineau CA, Tamblyn R, Feldman DE, Clarke AE. A population-based assessment of systemic lupus erythematosus incidence and prevalence--results and implications of using administrative data for epidemiological studies. Rheumatology (Oxford). 2007;46(12):1814-8. doi: 10.1093/rheumatology/kem233.

40. Bernatsky S, Ramsey-Goldman R, Clarke AE. Malignancy in systemic lupus erythematosus: what have we learned? Best Pract Res Clin Rheumatol. 2009;23(4):539-47. doi: 10.1016/j. berh.2008.12.007.

41. Hsu CY, Lin MS, Su YJ, Cheng TT, Lin YS, Chen YC, et al. Cumulative immunosuppressant exposure is associated with diversified cancer risk among 14832 patients with systemic lupus erythematosus: a nested case-control study. Rheumatology (Oxford). 2017;56(4):620-8. doi: 10.1093/ rheumatology/kew457.

42. Abbruzzese JL, Andersen DK, Borrebaeck CAK, Chari ST, Costello E, Cruz-Monserrate Z, et al. The interface of pancreatic cancer with diabetes, obesity, and inflammation: research gaps and opportunities: summary of a national institute of diabetes and digestive and kidney diseases workshop. Pancreas. 2018;47(5):516-25. doi: 10.1097/MPA.0000000000001037.

43. de Vivar Chevez AR, Finke J, Bukowski R. The role of inflammation in kidney cancer. Adv Exp Med Biol. 2014;816:197-234. doi: 10.1007/978-3-0348-0837-8_9.

44. Michaud DS. Chronic inflammation and bladder cancer. Urol Oncol. 2007;25(3):260-8. doi: 10.1016/j. urolonc.2006.10.002.

45. Sfanos KS, De Marzo AM. Prostate cancer and inflammation: the evidence. Histopathology. 2012;60(1):199-215. doi: 10.1111/j.1365-2559.2011.04033.x.

46. Kinlen LJ. Malignancy in autoimmune diseases. J Autoimmu. 1992;5:363-71. doi: 10.1016/0896-8411(92)90055-u.

47. Caillard S, Dharnidharka V, Agodoa L, Bohen E, Abbott K. Posttransplant lymphoproliferative disorders after renal transplantation in the United States in era of modern immunosuppression. Transplantation. 2005;80(9):1233-43. doi: $\quad$ 10.1097/01.tp.0000179639.98338.39.

48. Chow SK, Looi LM, Loh CS, Yeap SS. Cyclophosphamideinduced transitional cell carcinoma of bladder in lupus nephritis. Intern Med J. 2002;32(3):114-6. doi: 10.1046/ j.1445-5994.2002.d01-29.x.

49. Ortiz A, Gonzalez-Parra E, Alvarez-Costa G, Egido J. Bladder cancer after cyclophosphamide therapy for lupus nephritis. Nephron. 1992;60(3):378-9. doi: 10.1159/000186790.

50. Thrasher JB, Miller GJ, Wettlaufer JN. Bladder leiomyosarcoma following cyclophosphamide therapy for lupus nephritis. J Urol. 1990;143(1):119-21.doi:10.1016/s0022-5347(17)39886-5.

51. Bernatsky S, Joseph L, Boivin JF, Gordon C, Urowitz M, Gladman D, et al. The relationship between cancer and medication exposures in systemic lupus erythaematosus: a case-cohort study. Ann Rheum Dis. 2008;67(1):74-9. doi: 10.1136/ard.2006.069039.

52. Buzzeo BD, Heisey DM, Messing EM. Bladder cancer in renal transplant recipients. Urology. 1997;50(4):525-8. doi: 10.1016/S0090-4295(97)00305-1.

53. Abu-Shakra M, Gladman DD, Urowitz MB. Malignancy in systemic lupus erythematosus. Arthritis Rheum. 1996;39(6):1050-4. doi: 10.1002/art.1780390625.

54. Ragnarsson O, Grondal G, Steinsson K. Risk of malignancy in an unselected cohort of Icelandic patients with systemic lupus erythematosus. Lupus. 2003;12(9):687-91. doi: 10.1191/0961203303lu443oa.

55. Tarr T, Gyorfy B, Szekanecz E, Bhattoa HP, Zeher M, Szegedi $G$, et al. Occurrence of malignancies in Hungarian patients with systemic lupus erythematosus: results from a single center. Ann N Y Acad Sci. 2007;1108:76-82. doi: 10.1196/ annals.1422.008.

56. Tallbacka KR, Pettersson T, Pukkala E. Increased incidence of cancer in systemic lupus erythematosus: a Finnish cohort study with more than 25 years of follow-up. Scand J Rheumatol. 2018;47(6):461-4. doi: 10.1080/03009742.2017.1384054. 